\title{
Low leukocyte glutamate dehydrogenase activity does not correlate with a particular type of multiple system atrophy
}

\author{
R C DUVOISIN, W J NICKLAS, V RITCHIE, J SAGE, S CHOKROVERTY
}

From the Department of Neurology, University of Medicine and Dentistry of New Jersey, Robert Wood Johnson Medical School, New Brunswick, NJ, USA

SUMMARY Leucocyte glutamate dehydrogenase (GDH) activity was measured in 26 normal control subjects, 20 patients with multiple system atrophy presenting features of either olivopontocerebellar atrophy or striatonigral degeneration and in a heterogenous group of 15 patients with spinocerebellar degenerations. A broad range of GDH activity was found in all three groups. Low activity failed to correlate with a specific clinical entity. Patients followed to post-mortem examination to date have demonstrated histological features of at least three distinct morbid entities. It is concluded, contrary to earlier reports including the authors', that low leukocyte GDH activity does not identify a particular type of multiple system atrophy.

Plaitakis, Nicklas and Desnick ${ }^{1}$ reported in 1979 finding reduced activity of leukocytic glutamate dehydrogenase (GDH) in three patients with "spinocerebellar degeneration". The activities in the three patients were $38 \%, 29 \%$ and $31 \%$ respectively of the mean value of 20 control subjects. The first patient was a 19 year old male with a cerebellar syndrome with extrapyramidal rigidity, amyotrophy and peripheral neuropathy who had previously been described by Kilroy et al. ${ }^{2}$ as a case of juvenile Parkinsonism. The other two patients were siblings with an adult onset ataxia clinically resembling olivopontocerebellar atrophy (OPCA) type IV of Konigsmark and Weiner. ${ }^{3}$

Plaitakis, et al'subsequently found low leukocyte GDH activity in 10 of 12 patients classified as OPCA. Mean activity in these patients was $47 \%$ of normal controls. In a later fuller account these authors reported reduction of mean GDH activity in 12 of 23 patients with multiple system atrophy (MSA) to $52 \%$ of the mean activity found in 26 healthy adult control subjects, many of them spouses of the patients studied. $^{5}$ In contrast, the mean GDH activity in 65 patients with other degenerative neurological disorders including Parkinson's disease, progressive supranuclear palsy, Huntington's disease, dominant

Address for reprint requests: Prof Duvoisin, Department of Neurology, UMDNJ-Robert Wood Johnson Medical School, One Robert Wood Johnson Place, New Brunswick, NJ 08903, USA.

Received 5 May 1988. Accepted 1 July 1988 cerebellar degenerations and in six patients with MSA associated with progressive autonomic failure was similar to that found in the normal controls. The clinical features in the patients with low GDH activity resembled those of adult onset OPCA of the DejerineThomas type. Some, however, presented a predominantly cerebellar syndrome and others had widespread severe amyotrophy.

Duvoisin et $\mathrm{l}^{6}$ reported in 1983 a mean reduction of leukocyte GDH activity to less than $40 \%$ of control values in nine of 17 patients classified by clinical and radiological criteria as OPCA. Adding another patient to their series, Duvoisin and Chokroverty later reviewed the clinical features observed in 10 patients with low GDH activity. ' Their patients resembled those described by Plaitakas et $a^{5}$ with the addition of mild degrees of supranuclear ophthalmoplegia and a mild peripheral neuropathy documented in extensive neurophysiological studies. None had dysautonomia. Chokroverty et $a l^{8}$ failed to find evidence of neuropathy in comparable patients with normal GDH activity.

Two groups of Japanese workers have also reported reduced GDH activity in OPCA patients, but the reductions were minor. "Variable" reduction in GDH activity in leukocytes and a $30 \%$ reduction in platelets was found by Yamaguchi $e t$ al $^{9}$ in four of five patients classified as sporadic OPCA and in four patients with the Menzel type of dominantly inherited OPCA. There appeared to be a correlation between the severity of 
the clinical manifestations and platelet GDH activity. The authors concluded that low platelet GDH activity might be "quite specific" for OPCA. More recently, Konagaya et al $^{10}$ found a mean reduction of total leukocyte GDH activity in a series of 12 patients classified as OPCA to only $77.7 \%$ of control values.

Sorbi $e t$ al $^{11}$ also reported reduced GDH activity in platelets to a mean of $44 \%$ of control values in three of four patients with sporadic OPCA. They also found a more marked reduction in two affected members of a family with dominant OPCA. In another dominant OPCA family they found a different abnormality of GDH: the enzyme was not activated by ADP.

The suggestion arising from these data that defects in GDH might be associated with some forms of multiple system atrophy was rendered attractive by the finding of Plaitakis et $a l^{\prime \prime}$ of low fasting plasma glutamate tolerance in six patients with low leukocyte GDH activity. It was postulated that as a result of deficiency in this enzyme, the neuroexcitotoxin glutamate might accumulate in toxic amounts in the nervous system. ${ }^{1213}$ Apparent clinical benefit from feeding patients a diet high in leucine,${ }^{814}$ an amino acid known to activate the enzyme, gave further support to the concept of GDH deficiency as a clinical entity.

However, the diverse findings of the different groups of investigators, the fact that the loss of enzyme activity was only partial, the clinical heterogeneity noted in patients with low GDH activity, and the declining proportion of patients found to be GDH deficient on further experience has given rise to doubts regarding the significance of low GDH activity. Moreover, follow up of our early GDH-deficient patients has revealed diverse pathologies.

Plaitakis $e t a P$ reported that the reduction in GDH activity was limited to loss of a thermolabile fraction of GDH which corresponded to a "particulate" fraction ${ }^{5}$ separated by centrifugation. Konayaga et al ${ }^{10}$ also found evidence of a thermolabile particulate fraction in the leukocyte enzyme. These workers found a reduction of "total" GDH activity to only $77.7 \%$ of control values in their OPCA patients but a much larger reduction of the thermolabile particulate fraction to $26.8 \%$ of control values with no reduction in the thermostable solution fraction. These findings have complicated the subject and led to the hypothesis that there may be isoenzymes of GDH, a view disputed by others. The relationship of patients with small reductions in GDH activity to those reported earlier with large reductions has been uncertain.

In view of the potential importance of an identifiable defect of an enzyme regulating an excitotoxic neurotransmitter for our understanding of the aetiology and pathogenesis of the multiple system atrophies, further studies appeared necessary. Accordingly, we undertook a fresh survey of patients and normal controls, measuring the enzyme in both leukocytes and platelets with an improved assay procedure.

\section{Methods}

Patients. A total of 35 consecutive patients presenting with adult onset ataxia or atypical Parkinsonism were studied. Twenty-six spouse and other accompanying persons of comparable age served as normal controls. To assure uniformity, blood was drawn from most of the control subjects on the same date.

The patients were classified for the purpose of the study into two major groups. The first comprised 20 patients clinically diagnosed as striatonigral degeneration or olivopontocerebellar atrophy. In agreement with Oppenheimer's views ${ }^{15}$ these were combined under the rubric "multiple system atrophy" (MSA). These patients presented a combination of cerebellar and extrapyrimidal manifestations similar to those observed in our previous reports. ${ }^{78}$ None had dysautonomia. The second group who served as disease controls presented adult onset familial and sporadic cerebellar or spinocerebellar degenerations of unknown cause without extrapyramidal system involvement. The group included a patient with a cerebellar syndrome due to a familial leukodystrophy and a case of corticobasal degeneration recognised by the features described by Rebeiz et $a l^{16}$ and more recently by Watts et $a l^{17}$ and others. ${ }^{18}{ }^{19}$ This patient developed an asymmetric syndrome of progressive rigidity, apraxia, involuntary movements and dementia. Marked grasping reflexes and extensor plantar responses were found on examination. Brain CT and MRI studies documented a corresponding asymmetric cortical atrophy most prominent in the frontal and parietal areas.

Leukocyte preparation. Leukocytes were prepared from patients and control subjects by a modification of previouslypublished methods (Plaitakis et al, ${ }^{5}$ Duvoisin et al $)$. Briefly, $20 \mathrm{ml}$ of venous blood was collected in a $30 \mathrm{ml}$ syringe which had been previously wet with heparin. To the syringe was added $5 \mathrm{ml} \mathrm{6 \%}$ (w/v) Dextran 70, Pharmacia Macrodex, and the contents mixed and allowed to separate for one hour. The supernatant was collected and centrifuged for 10 minutes at $100 \mathrm{~g}$ in a refrigerated centrifuge (Dupont Sorvall RC-5B centrifuge). The resulting pellet was rinsed (without disturbing the pellet) with $1 \mathrm{ml}$ suspension medium $(0 \cdot 154 \mathrm{M} \mathrm{NaCl}$, 2.5 mM EDTA, pH 7.2). The pellet was then resuspended in $0.5 \mathrm{mg}$ room temperature suspension medium: $8 \mathrm{mg}$ of $0.87 \%(w / v)$ ammonium chloride was then added to lyse contaminating erythrocytes. Exactly $5 \mathrm{~min}$ later, the mixture was centrifuged at $100 \mathrm{~g}$ for $10 \mathrm{~min}$ in a refrigerated centrifuge. The pellet was rinsed with ice-cold suspension medium and then resuspended in the same medium. After centrifugation as above, the procedure was repeated. The final pellet was stored at -80 degrees until assay.

Glutamate dehydrogenase assay Aliquots of the above samples were assayed for GDH activity in a medium containing $50 \mathrm{mM}$ triethanolamine, $0.1 \mathrm{mM}$ dithiothreitol, $100 \mathrm{mM}$ ammonium acetate, $8 \mathrm{mM}$ 2-oxoglutarate, 2.6 mMEDTA, $2.5 \mu \mathrm{M}$ rotenone, 0 or $1 \mathrm{mM}$ ADP, $100 \mu \mathrm{M}$ NADH, final pH 8.0. Measurement was initiated by addition of the oxoglutarate and NADH disappearance was followed either by fluorimetry or spectrophotometry. ${ }^{6}$ In some cases 
$25 \mu \mathrm{M}$ guanosine triphosphate, a potent inhibitor of $\mathrm{GDH},{ }^{5}$ was added to ensure that GDH activity was being measured. Results were expressed in units; one unit equalled $1 \mu \mathrm{mol}$ NADH oxidised per hour per mg protein.

The "heat labile" GDH activity was measured by incubating homogenates at $47.5^{\circ} \mathrm{C}$ for 45 minutes essentially as described by Plaitakis $e t a l^{p}$ and then assaying an aliquot of this treated material using the procedure described above.

\section{Results}

Clinical studies A broad range of values for total leukocyte GDH activity was found in three groups of subjects studied: normal controls, patients with multiple system atrophy and disease controls (fig). In leukocyte homogenates from 26 control, healthy donors the activity was 1.24 , (SD 0.34) units; in the 15 disease controls it was $1 \cdot 17$, (SD 0.25) units and in the concurrent series of 20 multiple system atrophy cases, the average activity was 1.00 (SD 0.29) units. The mean GDH activity for this group is slightly lower than the means for the other two groups; the differences are significant at $p<0.001$. There is, however, almost complete overlap in the range of values in all three groups.

The GDH values described here are the ADPstimulated activities. Basal activities (that measured in the absence of ADP) also showed no major differences between groups. Nor were there any differences in the "heat-labile" activities. The average loss of activity after incubation at $45^{\circ} \mathrm{C}$ for 45 minutes was $43.3 \%$ (SD 18.7 ), $37.7 \%$ (SD 13.5) and $47.8 \%$ (SD 21.6) for the normal controls, disease controls and the multiple system atrophy patients respectively. There was no correlation between the proportion of heat lability and the enzyme activity measured prior to incubation in the same sample.

Analysis of the MSA group revealed no consistent clinical features distinguishing patients with low GDH activity from those with high or intermediate activities. The two patients with the lowest values and the two with the highest values presented very similar clinical features. Several normal control subjects and disease controls had values less than $50 \%$ the control values. The patient with corticobasal degeneration had the lowest value among the disease controls. The next lowest value was found in the patient with familial leukodystrophy.

\section{Discussion}

Leukocyte GDH activity in grouped data was significantly though only slightly lower in our MSA patients than in our normal and disease controls. Possibly, this may have reflected the effect of drugs in several patients. We have previously called attention to the fact that certain drugs including salicylates,

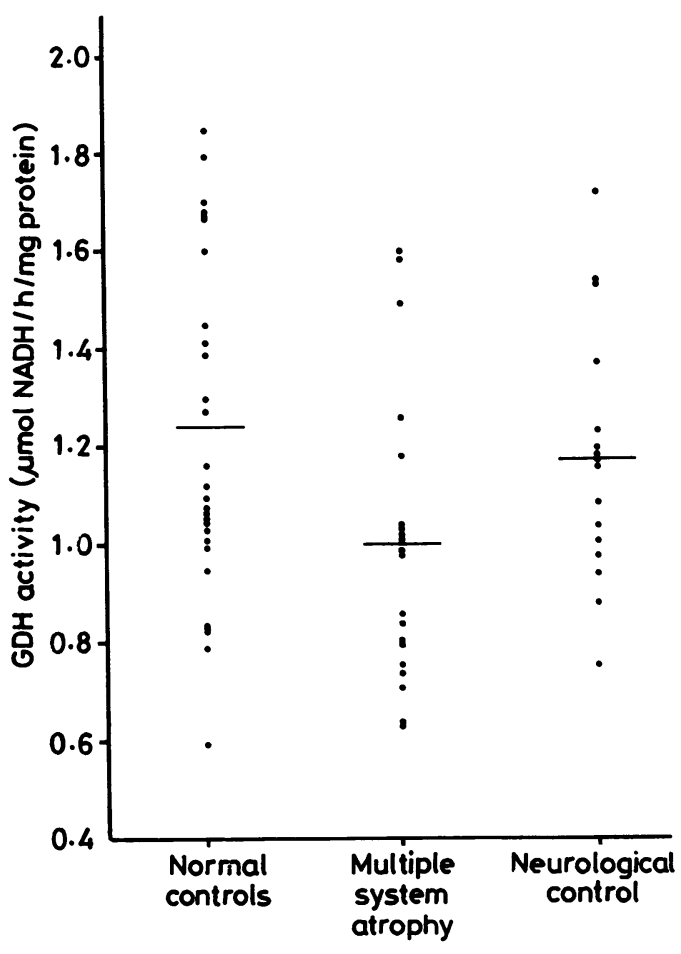

Figure Total glutamate dehydrogenase activity assayed in leukocytes obtained from venous blood in normal controls, patients with multiple system atrophy and in patients with a variety of spinocerebellar degenerations and other disorders.

indomethacin and dietary supplements containing linoleic and folic acids could produce depressed leukocyte GDH activity. ${ }^{7}$ There was no association of a loss of a "heat-labile" component of GDH activity with a specific type of multiple system atrophy.

We can reject the possibility that the group mean was depressed by a few patients with a particular disorder for several reasons. First, there was no correlation between clinical features and GDH activity. Second, low GDH activity was found in patients with different identifiable disorders. For example, one of the lowest values was found in the patient with corticobasal degeneration. Third, low GDH activity was also found in some normal control subjects.

Duvoisin and Chokroverty had found low GDH activity in some first degree relatives of their index cases but not in control subjects. ${ }^{7}$ They assumed that these individuals, though asymptomatic, were at risk for developing MSA at a later date and carried the presumed defective gene. However, in the present study, low GDH activity was found in some spouses and unrelated accompanying persons. It thus appears 
possible that variability in leukocyte GDH activity reflects an underlying polymorphism travelling independently through the population of patients and control subjects we have studied.

Postmortem observations Four patients found to have low GDH activity have been followed to necropsy. Case 1 of Plaitakis $e t$ al ${ }^{1}$ has recently been reported by Parker et $a l^{0}$ who found eosinophilic intranuclear neuronal inclusions of Cowdry Type A and $B$ throughout the cerebral cortex, thalamus, substantia nigra, locus ceruleus, inferior olivary nuclei, anterior horn cells and myenteric ganglia, findings typical of neuronal intranuclear hyaline inclusion disease. Another patient, case 2 in the report of Duvoisin et al, ${ }^{6}$ was found at necropsy to have widespread lipofuscinosis of the central nervous system. ${ }^{21}$ A third, reported by Plaitakis and Smith ${ }^{22}$ presented features typical of the Dejerine-Thomas type of OPCA. In a fourth patient who was included in the series of Duvoisin et at the predominant anatomical feature was widespread motor neuron degeneration with minimal associated cerebellar atrophy.

The diverse pathological findings in these patients leave little doubt that they represent at least three and probably four distinct morbid entities which could not reasonably be considered to share a common pathogenesis. We are therefore compelled on both clinical and pathological grounds to reconsider the suggestion derived from earlier work including our own that a systemic defect in glutamate regulation might be a marker for or play a pathogenetic role in a particular form of multiple system atrophy. The data now available show that low activity of the enzyme GDH does not correlate with a specific morbid entity or even a particular clinical syndrome.

\section{References}

1 Plaitakis A, Nicklas WJ, Desnick RJ. Glutamate dehydrogenase deficiency in three patients with spinocerebellar syndrome. Ann Neurol 1980;7:297-303.

2 Kilroy AW, Paulsen WA, Fenichel GM. Juvenile parkinsonism treated with levodopa. Arch Neurol 1972;27:350.

3 Konigsmark BW, Weiner LP. The olivopontocerebellar atrophies; a review. Medicine (Baltimore) 1970;49:227-41.

4 Plaitakis A, Berl S, Nicklas WJ, Yahr MD. Glutamate dehydrogenase deficiency in spinocerebellar degenerations: corrrelation with adult-onset recessive ataxia. Trans Am Neurol Assoc 1980;110:476-8.

5 Plaitakis A, Berl S, Yahr MD. Neurological disorders associated with deficiency of glutamate dehydrogenase. Ann Neurol 1984;15:144-53.

6 Duvoisin RC, Chokroverty S, Lepore F, Nicklas W. Glutamate dehydrogenase deficiency in patients with olivopontocerebellar atrophy. Neurology 1983;33:1322-6.

7 Duvoisin RC, Chokroverty S. Clinical expression of glutamate dehydrogenase deficiency. In: Duvoisin R C, Plaitakis A, eds. The Olivopontocerebellar Atrophies, Adv Neurol, New York: Raven Press 1984;40:267-79.

8 Chokroverty S, Duvoisin RC, Sachdeo R, Sage J, Lepore F, Nicklas W. Neurophysiologic study of olivopontocerebellar atrophy with or without glutamate dehydrogense deficiency. Neurology 1985;35:652-9.

9 Yamaguchi T, Hayashi K, Murakami H, Ota K, Maruyama S. Glutamate dehydrogenase deficiency in spinocerebellar degenerations. Neurochem Res 1982;7:627-36.

10 Konagaya Y, Konayaga M, Mano Y, Takayanagi T. Glutamate dehydrogenase and its isoenzyme activity in olivopontocerebellar atrophy. J Neurol Sci 1986;74:231-6.

11 Sorbi S, Tonini S, Giannini E, Piacenti S, Marinin P, Amaducci L. Abnormal platelet glutamate dehydrogenase activity and activation in dominant and nondominant olivopontocerebellar atrophy. Ann Neurol 1986;19:239-45.

12 Plaitakis A, Berl S, Yahr MD. Abnormal glutamate metabolism in an adult-onset degenerative neurological disorder. Science 1982;216:193-6.

13 Plaitakis A. The olivopontocerebellar atrophies. Semin Neurol 1982;2:334-42.

14 Plaitakis A, Berl S, Yahr MD. The treatment of GDH-deficient olivopontocerebellar atrophy with branched chain amino acids. Neurology 983;33:9(suppl 2) 78.

15 Oppenheimer DR. Diseases of the basal ganglia, cerebellum and motor neurons. In: Adams JH, Corsellis JAN, Duchen LW, eds. Greenfield's Neuropathology, 4th ed. London: Edward Arnold, 1984;699-747.

16 Rebeiz JJ, Kolodny EH, Richardson EP. Corticodentatonigral degeneration with neuronal achromasia. Arch Neurol 1968;18:20-23.

17 Watts RL, Williams S, Growdon JD, Young RR, Haley Jr EC, Beal MF. Corticobasal ganglionic degeneration [abstract]. Neurol 1985;35 (suppl 1): 178.

18 Monaco S, Sepic J, Cavalloro T, Ferrari S, Rizzuto N. Corticodentatonigral degeneration and amyotrophy with dystrophic achromatic neurons [abstract]. Ital J Neurol Sci 1987;8:193-4.

19 Case Records of the Massachusetts General Hospital, Case 38, 1985. N Eng J Med 1985;313:739-48.

20 Parker JC, Dyer ML, Paulsen WA. Neuronal intranuclear hyaline inclusion disease associated with premature coronary atherosclerosis. J Clin Neuro-opthalmol 1988;7:244-9.

21 Chokroverty S, Khedekar R, Derby B, et al. Pathology of olivopontocerebellar atrophy with glutamate dehydrogenase deficiency. Neurology 1984;34:1451-5.

22 Plaitakis A, Smith J. Biochemical and morphological changes of the brain in a patient dying of GDH-deficient olivopontocerebellar atrophy. Ann Neurol 1986;20:152A. 